\title{
A feature-weighting account of priming in conjunction search
}

\author{
STEFANIE I. BECKER \\ University of Queensland, St. Lucia, Australia \\ AND \\ GERNOT HORSTMANN \\ University of Bielefeld, Bielefeld, Germany
}

\begin{abstract}
Previous research on the priming effect in conjunction search has shown that repeating the target and distractor features across displays speeds mean response times but does not improve search efficiency: Repetitions do not reduce the set size effect - that is, the effect of the number of distractor items - but only modulate the intercept of the search function. In the present study, we investigated whether priming modulates search efficiency when a conjunctively defined target randomly changes between red and green. The results from an eyetracking experiment show that repeating the target across trials reduced the set size effect and, thus, did enhance search efficiency. Moreover, the probability of selecting the target as the first item in the display was higher when the target-distractor displays were repeated across trials than when they changed. Finally, red distractors were selected more frequently than green distractors when the previous target had been red (and vice versa). Taken together, these results indicate that priming in conjunction search modulates processes concerned with guiding attention to the target, by assigning more attentional weight to features sharing the previous target's color.
\end{abstract}

It is common knowledge that people cannot process and consciously perceive all information present in a visual scene. At any point in time, attention selects only some items, which are prioritized for perceptual processing. Given the importance of attention for perception and action, researchers have made great efforts to find out what guides attention. Probably the most influential research tool has been the visual search paradigm. In a typical visual search task, the observer has to search for a target among several other objects and to indicate the presence or the identity of the target with a manual response.

Previous research has suggested a dichotomy in search performance for tasks in which the target is defined by a single feature versus a conjunction of different features: For instance, when participants have to search for a red target among a group of homogeneously green targets ( pop-out search task), response times (RTs) are virtually independent of the number of distractor items or the overall set size (e.g., Treisman, 1982). In contrast, when the target differs only in a conjunction of features from the distractors- for instance, when a red vertical target has to be singled out from green vertical and red horizontal distractors - search times usually increase linearly with set size (e.g., Treisman, 1988; Treisman \& Souther, 1985). Searches in which RTs increase linearly with set size have been termed inefficient or serial, whereas searches in which the target can be found independently of the number of surrounding objects have been termed efficient or parallel.
The difference between efficient and inefficient search has been explained theoretically by a two-stage visual process (e.g., Itti \& Koch, 2000; Treisman \& Sato, 1990; Wolfe, 1994): According to this view, processes at the first, preattentive stage operate in parallel across the entire visual field and exmtract information about the position of single features. Since information about single features is available to the visual system at a very early stage of processing, attention can be immediately deployed to the search target if it possesses a unique feature. Deploying attention to an item in the visual field gates the passage of information to higher stages of processing, such as visual object recognition and response generation. Critically, processes at this second, attentive stage are capacity limited and integrate information from a limited part of the visual field in a time-consuming process. Thus, if the target does not differ from the distractors in a single feature, processes at the first stage of processing cannot single out the search target. This accounts for the finding of inefficient search for conjunction targets: When the target is defined by a specific conjunction of features, timeconsuming attentional processing is necessary to locally combine the information of the corresponding features, resulting in longer search times with an increasing number of distractor items.

Although past research has shown that conjunction searches mostly yield inefficient search performance, there has also been some evidence to the contrary: Some feature

S. I. Becker, s.becker@psy.uq.edu.au 
conjunctions can apparently be found efficiently - that is, with search times that are independent of the number of distractors. Efficient search for conjunctively defined targets can be demonstrated, for instance, for conjunctions of binocular disparity and movement, as well as for conjunctions of either color or motion with stereo depth (Nakayama \& Silverman, 1986), conjunctions of shape with a certain direction of movement (McLeod, Driver, \& Crisp, 1988), conjunctions of size and orientation and size and color (Wolfe, Cave, \& Franzel, 1989), and also multiple conjunction targets that consist of four or more possible feature combinations (Wang, Kristjánsson, \& Nakayama, 2005).

The finding that some conjunctions can be found independently of the set size at first seems to be incompatible with the basic tenet of two-stage visual search theories that only information about simple features is preattentively accessible. To reconcile the findings of efficient conjunction search with two-stage visual search theories, several mechanisms have been proposed. According to the proponents of two-stage visual search theories, inhibition of distractor features (Treisman \& Sato, 1990) or enhancement of target features (Wolfe et al., 1989) might account for efficient conjunction search. For instance, if the target is consistently colored red and of square shape, participants might be able to set their attentional control settings to these two features, so that items matching the description will receive the largest weights and can immediately guide attention to the target. Setting the attentional control settings to the target features can result either in inhibition of distractor features, such as all green-colored or round-shaped items, or in enhanced preactivation of the target features (but for alternative theories, see Duncan \& Humphreys, 1989; D'Zmura, 1991; Rauschenberger \& Yantis, 2006; Wang et al., 2005).

The present study will be concerned with yet another explanation: More recently, it has been proposed that efficient conjunction search could be due to intertrial priming effects (e.g., Geyer, Müller, \& Krummenacher, 2006).

\section{Intertrial Priming Effects in Conjunction Search}

Feature-priming effects were first discovered in a popout search task, in which participants had to search for a target that could be either green or red, while all of the distractors assumed the opposite color. The results showed that RTs were shorter when the target and the distractor features were the same as in the previous, $n-1$ trial than when the assignment of colors switched, as compared with the previous trial (Maljkovic \& Nakayama, 1994). In the pioneering study of Maljkovic and Nakayama (1994), this intertrial effect pertained only to the target-defining feature and was not related to the response: The response-indicative feature was constituted by the target's shape, and repeating the target shape, in turn, did not result in any facilitation. This led Maljkovic and Nakayama (1994) to propose that intertrial contingencies do not modulate response-related processes but, instead, affect processes that guide attention to the target: According to their priming of pop-out hypothesis, repeating the target enhances the pop-out effect of this feature in subsequent trials, which increases its capability to summon attention to its position (Maljkovic \& Nakayama, 1994, 1996). On this view, selection of the target entails enhancing the activation signals of the target feature and/or decreasing the activation signals of the distractor feature, which carry over to the next trial and lead to priming (Maljkovic \& Nakayama, 1994, 1996). Originally, carryover effects of activation and inhibition patterns were conceptualized as charges that increase the attentiondriving capacity of single features in the fashion of a parallel resistive-capacitance configuration (Maljkovic \& Nakayama, 1996). However, it is also possible to conceptualize priming in terms of a feature-weighting mechanism (e.g., Leonard \& Egeth, 2008; see also Found \& Müller, 1996; Kristjánsson, 2006a; Müller, Heller, \& Ziegler, 1995). On this view, feature weights or the attention-driving capacity of each feature in the display is adapted on a trial-by-trial basis. Thus, switch costs are basically due to the fact that, on switch trials, the distractor features are prioritized for attentional selection, which leads to erroneous attention shifts to one of the distractor items. On the feature-weighting account, activation of previously attended areas in the visual field is supposed to help to reorient attention and the gaze to previously inspected areas or features.

Priming effects could also be observed in conjunction search tasks, in which the target was defined by a conjunction of color and orientation or a conjunction of color and texture. In three studies, responses were found to be much faster when the target and distractor features from the previous trial were repeated than when they changed (Geyer et al., 2006; Hillstrom, 2000; Kristjánsson, Wang, \& Nakayama, 2002). This demonstrates that priming modulates search performance even in inefficient conjunction search tasks, in which search times linearly increase with the number of distractors. The authors interpreted their findings as support for the view that repeating the target and distractor features across displays facilitates early attentional or preattentive perceptual processes, by enabling more efficient perceptual grouping of the distractors (Kristjánsson, 2006a; Kristjánsson et al., 2002) or by speeding the processing of all display elements in parallel (Geyer et al., 2006).

However, it may also be doubted whether the results can be interpreted in such a way that priming facilitates the deployment of attention to the target. It should be observed that search efficiency is usually conceptualized as a function of set size, which is the number of distractor items present in the display. More precisely, search efficiency is defined by the search rate or the slope of the function relating RT to set size - that is, the increase in search time, as indicated by the manual RTs, with each added distractor item (expressed in milliseconds/item). Commonly, search performance is labeled efficient only if the corresponding search rates either do not differ significantly from zero (e.g., Yantis \& Egeth, 1999) or are at least below $10 \mathrm{msec} /$ item (Treisman \& Sato, 1990; Wolfe, 1998; Wolfe, Friedman-Hill, Stewart, \& O'Connell, 1992). In line with this criterion, previous studies have cited search rates of around $10 \mathrm{msec} / \mathrm{item}$ as evidence for efficient conjunction search (McLeod et al., 1988; Nakayama \& Silverman, 1986; Treisman \& Sato, 1990; Wang et al., 2005; Wolfe et al., 1989).

In contrast to this, previous studies investigating priming effects in conjunction search have failed to show a 
reduction of the set size effect. In Hillstrom's (2000) study, participants searched for a red textured or pink nontextured target among four, six, or eight red nontextured and pink textured distractors of various orientations. The results showed that repeating the target and distractor features across displays speeded mean RTs by about $440 \mathrm{msec}$ but did not affect the search slope, which was consistently around $27 \mathrm{msec} /$ item. Similarly, in Kristjánsson et al.'s (2002) study, six to eight repetitions of the target and distractor features speeded mean RTs by about $100 \mathrm{msec}$. Again, however, search rates were "constant under all conditions," which led Kristjánsson et al. to conclude that "The priming benefits are independent of the set size on a particular trial" (p. 49). Finally, in the study of Geyer et al. (2006), repeating the target and distractor features speeded mean RTs by about $40 \mathrm{msec}$, as compared with complete changes of the target and distractor features. However, as in Kristjánsson et al., the search slope remained unaffected by priming. Correspondingly, Geyer et al. concluded that the results of their study and previous studies together indicated that "repetition does not affect the search time per element (the slope of the RT/display size function), but rather the base RTs ( $y$-intercept of the function)" (p. 748).

Thus, previous results do not warrant the conclusion that repeating the target and distractor features across displays in conjunction search generally improves search efficiency in a manner that could account for efficient conjunction search. Since none of the previous studies showed that priming leads to a reduction of the set size effect, the claim that repetitions of target and distractor features might have improved search efficiency in previous conjunction search studies in such a way as to yield efficient conjunction search (with search rates below $10 \mathrm{msec} /$ item) is unwarranted.

\section{Three Explanations of the Priming Effect in Conjunction Search}

The failure to demonstrate increases in search efficiency by priming also leads to more serious problems, because it seems to be incompatible with the more general claim that priming facilitates attention shifts to the target. As Kristjánsson et al. (2002) noted, "The lack of any change in search rate is unexpected in the context of an expected increase in target identifiability" (p. 49).

Correspondingly, a first and quite damaging explanation for the failure to observe modulations in the set size effect by priming would be to claim that priming in conjunction search only speeds processes unrelated to the search process. As Kunar, Flusberg, Horowitz, and Wolfe (2007) argued with respect to the contextual cuing effect, observing only effects in the base RTs or in the $y$-intercept of the search function suggests that repeated presentations of a search display facilitate processes unrelated to processes of attentional guidance, such as decision-related or response selection processes.

If this postselectional view is correct, priming effects in conjunction search will differ from priming in pop-out search search tasks. Several studies have demonstrated that in pop-out search, repeating the target-distractor features across displays speeds attention shifts and eye movements to the target (Becker, 2007, 2008a, 2008b, 2008c; Goolsby \& Suzuki, 2001; McPeek, Maljkovic, \& Nakayama, 1999) but does not affect processes unrelated to attentional guidance (Becker, 2008b, 2008c; Olivers \& Humphreys, 2003). Thus, if the priming effect in conjunction search is indeed based on postselectional processes, priming effects in conjunction search will not be identical to priming of pop-out and cannot help reorient attention or the gaze to previously attended items.

Several different mechanisms have been proposed to reconcile an attentional account of the priming effect with the failure to observe modulations in search efficiency. According to Geyer et al. (2006), priming modulates processes concerned with parallel encoding of all target and distractor features. Similarly, Kristjánsson et al. (2002) proposed that search efficiency is generally determined by the number of structural units present in the display and that repetitions facilitate these grouping processes (Wang et al., 2005; see also Duncan \& Humphreys, 1989). Finally, Hillstrom (2000) proposed an episodic retrieval account of the priming effect. On this view, repeating the targetdistractor features across displays speeds the retrieval of memory traces that assign attentional priorities to all display items. Within the framework of two-stage theories of visual attention (which, however, are not endorsed by all of the above-mentioned authors), priming thus does not directly affect how attention is deployed to the items in the search display. Instead, repetitions of the target and distractor features speed preattentive processes that must be completed before attention can be allocated to any item in the search display. These processes do not directly change the probability of selecting either the target or one of the distractors (or groups of distractors), or the attentional priorities assigned to each feature on a trial-by-trial basis, as proposed by a feature-weighting view. Instead, priming merely modulates the time course of selection, or the speed of prioritizing elements in the display, without changing the probabilities that individual items will capture attention (cf. Hillstrom, 2000). Hence, all of the above-mentioned accounts can explain why priming affects only the baseline RTs without modulating the set size effect, while simultaneously maintaining that priming does not exert its effects by modulating postselectional processes.

A third possibility would be to maintain that priming usually affects the visual selection process, by modulating the probabilities of selecting the target versus the distractor feature, but that this effect went undetected in previous studies, either because the RT $\times$ set size function is not the most sensitive or most reliable indicator for attentional processes (as compared with eye movements, for example) or because previous studies were not optimally designed to detect variations in the slope of the search function. The RT $\times$ set size function might not be the most sensitive measure for attentional processes, because manual responses generally include a variety of different processes - notably, decisional processes and response selection processes - which are usually classified as postselectional processes. Although it is generally assumed that the RT $\times$ set size function reflects the frequency with which distractors are selected prior to 
target selection (as a function of the number of distractors), differences in the set size also affect the decision criterion for making a response (statistical decision noise; Huang \& Pashler, 2005; Palmer, 1995). Since the RT $\times$ set size function can be determined by a conglomerate of different processes, it is possible that small changes in the probability of selecting the target feature or the distractor feature are not accompanied by changes in the search slope. In contrast, monitoring eye movements probably allows more direct and reliable observations about changes in search efficiency, because eye movements are usually preceded by covert attention shifts and are less contaminated by postselectional processes (e.g., Deubel \& Schneider, 1996).

Alternatively or additionally, it is also conceivable that priming usually modulates search efficiency and the slope of the search function but that this effect went undetected in previous studies. First, previous studies were possibly not ideally designed to study processes that guide attention to the target, because the target was not consistently defined by a fixed set of features. In two studies, participants had to search for a red horizontal or vertical bar among red distractors of the opposite direction and green distractors that had the same orientation as the target. Hence, the target was defined not by a conjunction of fixed, predefined features but, instead, by being the only red item in the display with a deviating orientation (Geyer et al., 2006; Kristjánsson et al., 2002). To be certain whether a selected item was indeed the target, participants in this task presumably had to compare the features of the target with the features of the distractors, which, in turn, may have attenuated the effects of more versus less efficient initial visual selection of the target-for instance, by priming.

Second, it is possible that the participants in previous studies engaged in a subset search strategy. The capability to search a specifically colored subset of items has been demonstrated in various studies (e.g., Egeth, Virzi, \& Garbart, 1984; Kaptein, Theeuwes, \& van der Heijden, 1995) and might have occurred in all of the studies cited above: in the studies of Geyer et al. (2006) and Kristjánsson et al. (2002) because the target was consistently a red-colored item presented among red- and green-colored distractor items, and in the study of Hillstrom (2000) because the target color was validly precued before the onset of the search display and varied in a predictable sequence of AABBAABB (i.e., two red targets were always followed by two pink targets, which were followed by two red targets, etc.). Given that the target color was always known beforehand, it is possible that the participants in these studies restricted search to the relevant subset of items that contained the target by attending only to items that possessed the target color, whereas they mostly ignored items of the other color.

Such a subset search strategy usually results in a reduction of the relevant or effective set size (e.g., Palmer, 1995). For instance, when participants are instructed to restrict search to a subgroup of red-colored items, search is slowed only when the set size of the red-colored distractors is increased, whereas increasing the set size of the green distractor items does not affect search performance. (Egeth et al., 1984; Kaptein et al., 1995). In previous stud- ies on the priming effect, a subset search strategy might have compromised detecting an effect of priming on the search slope, especially if displays did not always contain an equal number of different distractors in each subset, and the relevant set size varied within each set size condition so that, for example, the set size 8 condition could include 3,4 , or 5 distractors of the same color as the target. ${ }^{1}$ Apart from that, it is possible that only some participants engaged in a subset search strategy, and probably merely on a certain portion of trials, whereas other participants followed a different strategy. The possible variation both in the physical set size and in the chosen strategy would, however, have led to large variations in the individual slopes of the search function, which would have made it difficult to detect possible priming effects on the slope. Consequently, it is possible that priming did reduce the effect of set size in previous studies but that this went unnoticed because there was too much variance in the search slopes within and across individual participants.

\section{Aims of the Present Study}

The first aim of the present study was to critically test whether repeating the target-distractor features across displays in conjunction search affects the search process, as was claimed by Kristjánsson et al. (2002), Hillstrom (2000), and Geyer et al. (2006), or whether it predominantly modulates processes after the target has been found, as has been proposed by the postselectional view. Supposing that priming modulates processes concerned with the search process, the second aim was to gain new insights about the mechanism that instigates repetition facilitation effects and switch costs. More precisely, we wanted to investigate whether priming in conjunction search affects processes that guide attention, in accord with a feature-weighting mechanism, or whether priming rather modulates preparatory processes (e.g., memory retrieval, grouping, or parallel encoding), which operate before attention is deployed to any item in the display (Geyer et al., 2006; Hillstrom, 2000; Kristjánsson et al., 2002).

To that end, the present conjunction search task was designed so as to eliminate the advantage of subset search, by randomly changing the target colors between red and green. Moreover, the displays always contained an equal number of red and green items, allowing assessment of reductions in search slope even when observers engaged in a subset search strategy. Second, the present experiment included both RT and eye movement recordings. As was argued above, eye movements may provide a more sensitive or more reliable measure for changes in search efficiency. Thus, comparing effects on eye movements and on the slopes of the search function may help us to decide whether the RT $\times$ set size function can reliably indicate changes in search efficiency.

Moreover, eye movements allow a more direct test of the mechanism underlying priming. On the featureweighting view, priming should change the weights of the possible target-defining features on a trial-by-trial basis. Thus, trials on which the target is colored red should lead to more frequent selections of red-colored distractors on subsequent trials, whereas green-colored distractors 
should be selected less frequently. On the other hand, on the episodic retrieval account and the alternative views, priming should not change the probability that a certain feature will be selected on a trial-by-trial basis but should only modulate the time course needed to select the target (e.g., by modulating the time course needed to retrieve a prioritization rule or the time needed to encode or group stimuli).

\section{Experiment}

In the experiment, participants had to search for a conjunctively defined target that could be either a red horizontal bar presented among red vertical and green horizontal distractors or a target that was a green vertical bar presented among red vertical and green horizontal distractor items. Targets of two different colors and orientations were chosen to exclude the possibility that the target could be found by attending to a certain subset of items. To determine search efficiency and to detect possible effects of priming on search efficiency, the set size was varied randomly among 4, 8, and 16 items. Moreover, to ensure that the participants had to move their eyes during search, the target was present on all trials, and the participants had to respond to small items located inside the target (compound search task).

A first aim of the experiment was to test whether priming modulates the time needed to find the target or affects postselectional processes commencing after the target has been found. If repeating the target-distractor features in conjunction search affects the search process, we would expect that the target should be visually selected earlier on repetition trials than on switch trials: That is, the target fixation latencies or visual response times (VRTs) should be shorter on repetition trials than when the features of target and distractors from the previous trial change. In contrast, if priming modulates only postselectional processes unrelated to the "search proper," priming should affect only processes that occur after the search target has been found. Thus, repeating the target and distractor features could affect the target fixation durations - that is, the duration the eyes remain fixated on the target after selection.

The second aim of the present experiment was to determine whether priming affects how attention is deployed to different items or modulates early perceptual processes that do not play an active role in guiding attention to the target. If priming changes the attentional weights of individual features on a trial-by-trial basis, repeating the target-distractor features across trials should lead to a higher probability of selecting the target as the first item in the display, which in turn should lead to a higher percentage of first fixations on the target on repetition trials than on switch trials. Alternatively, if priming affects only the time course of early perceptual processes without directly affecting the attentional weights of individual features, priming should modulate only the latencies of the first eye movement, so that repeating the target-distractor features lead to shorter saccade latencies of first saccades directed to the target. However, such a result pattern would also be in accord with the feature-weighting view and, thus, can- not decide the question of whether priming speeds attention shifts to the target or preparatory processes that have to be completed before the attention shift.

To further determine whether priming accords with a feature-weighting mechanism, the mean number of fixations on green and red distractor items and the respective fixation durations were analyzed separately. If distractors that have the same color as the target on the previous trial are selected more frequently than distractors of the other color, priming apparently operates on a feature-weighting mechanism. On the other hand, if intertrial contingencies merely modulate the time course of retrieving a priority rule, or other preparatory processes concerned with stimulus encoding or grouping, there should be no preference for distractors that have the same color as the previous target.

Finally, to assess whether the RT $\times$ set size function can reliably indicate changes in search efficiency, we additionally measured the distractor fixation durationsthat is, the duration the eyes remain fixated on a distractor item. Note that the slope of the search function allows reliable inferences about the mean number or frequency of distractor selections only if the time needed to reject each distractor remains constant across different set size conditions (because the slope of the search function is determined both by the mean number of distractors that are selected before target selection and by the time needed to reject each distractor). ${ }^{2}$

\section{METHOD}

\section{Participants}

Eight students from the University of Bielefeld, Germany, took part in the experiment as paid volunteers $(€ 6 / \mathrm{h})$. Three of them were male, 5 female, and they had a mean age of 23.6 years. All the participants had normal or corrected-to-normal vision and were naive as to the purpose of the experiment.

\section{Materials}

An Intel Pentium 4 CPU 3.00-GHz computer with a 19-in. SVGA color monitor (AOC) controlled the timing of events and generated the stimuli. The stimuli were presented with a resolution of $1,024 \times$ 768 pixels and a refresh rate of $99.9 \mathrm{~Hz}$. For recording of eye movements, a video-based infrared eye-tracking system (iViewX, SMI, Teltow, Germany) with a spatial resolution of $0.1^{\circ}$ and a temporal resolution of $240 \mathrm{~Hz}$ was used. The participants were seated in a dimly lit room, with their head fixated by the eyetracker's chinrest and forehead support, and viewed the screen from a distance of $92 \mathrm{~cm}$. For registration of manual responses, a standard USB optical mouse was used. Event scheduling and RT measurement were controlled by the Presentation software (Neurobehavioral Systems).

\section{Stimuli}

The response-indicative stimuli consisted of black arrowhead stimuli, " $<$ " and " $>$ " (Arial Black, font size $=13$ pt.), which were kept small $\left(0.12^{\circ} \times 0.12^{\circ}\right)$ in order to induce fixations on them for discrimination. The response-indicative stimuli were located in the center of red- or green-colored bars, which were matched for luminance $\left(1.9 \mathrm{~cd} / \mathrm{m}^{2}\right.$; MAVOLUX digital photometer) and were oriented either horizontally $\left(1.9^{\circ} \times 0.5^{\circ}\right)$ or vertically $\left(0.5^{\circ} \times 1.9^{\circ}\right)$. All the stimuli were presented on the positions of a $4 \times 4$ grid, and each stimulus was located at a distance of $3.4^{\circ}$ horizontally and vertically from the next item, center to center. The most outward stimuli were presented $6.2^{\circ}$ away from the vertical frames of the display and $3.7^{\circ}$ away from the horizontal frames. The target could be either a red horizontal bar presented among green horizontal and red verti- 

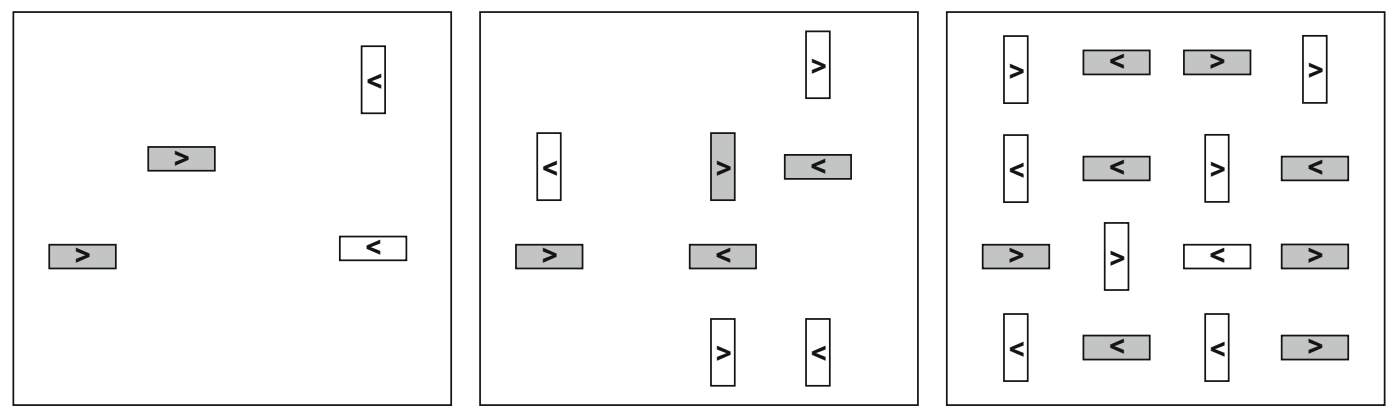

Figure 1. Example of the stimulus displays, depicted separately for the set size 4, 8, and 16 conditions. The target could be either red and horizontal or green and vertical, and participants responded to the " $>$ " and "<" stimuli by pressing a button. White-colored bars represent red stimuli, and the gray-colored bars represent the green stimuli. The stimuli are not drawn to scale; all the stimuli, particularly the response-indicative " $>$ " and " $<$ " items, were much smaller.

cal distractors or a green vertical bar presented among red vertical and green horizontal distractor bars. All the stimuli were presented against a constantly white background $\left(7.2 \mathrm{~cd} / \mathrm{m}^{2}\right)$. Figure 1 depicts an example of the stimuli in each set size condition.

\section{Design}

The experiment consisted of the within-subjects conditions of set size, intertrial contingencies of the target-defining feature, and intertrial contingencies of the response-indicative feature. Concerning the intertrial contingencies, the target and distractor items were randomly drawn on each trial. Thus, the target features on a current trial $n$ could be the same as those on the previous, $n-1$ trial, constituting a same target feature trial, or they could differ from those of the previously presented target, representing a different target feature trial. In addition, the response-related items could also be repeated, constituting a response repetition trial, or the response-related items could differ from those on the previous, $n-1$ trial, constituting a different-response trial. The probability for target or response repetitions was .5 throughout the experiment.

The position of the target and distractors, as well as the orientation of the response-indicative item inside the target, were drawn randomly, with the restriction that each display contained an equal number of red- and green-colored stimuli: For instance, in the set size 16 condition, if the target was the red horizontal bar, 7 red vertical distractors and 8 green horizontal distractors were added to the display. In addition, the number of the response-indicative stimuli was controlled so that each display contained an even number of leftand right-pointing arrowhead stimuli. Before the beginning of the experiment, the participants completed 20 practice trials that were not recorded, followed by 480 experimental trials. The participants were allowed a short rest after completing 160 trials.

\section{Procedure}

Each trial started with the presentation of a small black fixation cross. The participants were instructed to fixate on the center of the cross. At the beginning of each trial, a fixation control was implemented: The stimulus display was presented only if the tracking was stable (no blinks) and the gaze was within 50 pixels $\left(1^{\circ}\right)$ of the center of the fixation cross, for at least $350 \mathrm{msec}$ (within a time window of 3,000 msec). Otherwise, the participants were calibrated anew (5-point calibration), and the next trial started again with the fixation control.

Upon presentation of the stimulus display, the participants were required to search the display for the target and to press the right mouse button if the response-indicative item was an arrowhead pointing to the right (">") and the left mouse button if it was pointing to the left (" <"). The stimulus display remained on screen until response and was immediately succeeded by a feedback display. The feedback consisted of the black printed words "right" or "wrong" (in German, $14 \mathrm{pt}$.), which were presented centrally and remained on screen for $500 \mathrm{msec}$. After an intertrial interval of $500 \mathrm{msec}$, in which a blank white screen was presented, the next trial started with the presentation of the fixation cross.

Before the experiment, the participants were calibrated with a 5 -point calibration and were given written instructions about their tasks. The participants were given no instructions concerning their eye movements but were instructed to respond to the target as quickly as possible without making mistakes. On average, it took $90 \mathrm{~min}$ to complete the experiment

\section{RESULTS}

Trial data were excluded when the manual response exceeded 3,000 msec or when the target had not been fixated within $3,000 \mathrm{msec}$ from the beginning of the trial. The eyes were counted as fixating on the target or on a distractor if the gaze was within a distance of $1^{\circ}$ from the center of the bar and no saccade occurred (velocity smaller than $30^{\circ} / \mathrm{sec}$ ). Removing the outliers resulted in a loss of $4.6 \%$ of the manual RT data and $12.0 \%$ of the eye position data.

All data were statistically analyzed by a $3 \times 2 \times 2$ ANOVA comprising the within-subjects variables of set size (4 vs. 8 vs. 16), feature priming (repeating the target and distractor features vs. changing the target-distractor features as compared with the previous, $n-1$ trial), and response priming (repeating the response vs. switching the response from the previous, $n-1$ trial). The $F$ values and $p$ values of the ANOVA results are shown in Table 1.

\section{Manual Responses}

The mean RTs and error scores in each intertrial condition are shown in Figure 2 and Table 2, respectively, separately for each feature priming $\times$ response priming condition for each of the corresponding set sizes: Error scores were quite low, averaging $2.2 \%$, and were significantly modulated only by response repetition, with fewer errors at response repetition trials $(M=2.2 \%)$ than at differentresponse trials $(M=2.3 \%)$. None of the remaining effects reached significance (see Tables 1 and 2).

As can be seen in Figure 2, RTs increase with increasing set size, resulting in a significant main effect of set size (see also Table 2), which is a hallmark of inefficient search. Moreover, Figure 2 indicates that trials on which 
Table 1

Results of the $3 \times 2 \times 2$ ANOVA, Comprising the Variables of Set Size (4, 8, or 16), $n-1$ Target Color (Same or Different Color as Previous Target), and $n-1$ Response-Indicative Feature (Same Response or Different Response Than in Previous Trial)

\begin{tabular}{|c|c|c|c|c|c|c|c|c|c|c|c|c|c|c|}
\hline & \multicolumn{2}{|c|}{ RT } & \multicolumn{2}{|c|}{ Error } & \multicolumn{2}{|c|}{$\begin{array}{c}\text { Target } \\
\text { Fixation } \\
\text { Latency }\end{array}$} & \multicolumn{2}{|c|}{$\begin{array}{c}\text { Percentage } \\
\text { of First } \\
\text { Saccades }\end{array}$} & \multicolumn{2}{|c|}{$\begin{array}{c}\text { Latency } \\
\text { of First } \\
\text { Saccade }\end{array}$} & \multicolumn{2}{|c|}{$\begin{array}{l}\text { Distractor } \\
\text { Fixation } \\
\text { Duration }\end{array}$} & \multicolumn{2}{|c|}{$\begin{array}{c}\text { Target } \\
\text { Fixation } \\
\text { Duration }\end{array}$} \\
\hline & $F$ & $p$ & $F$ & $p$ & $F$ & $p$ & $F$ & $p$ & $F$ & $p$ & $F$ & $p$ & $F$ & $p$ \\
\hline Set size (SS) & 61.6 & $<.001$ & $<1.0$ & .402 & 82.3 & $<.001$ & 90.3 & $<.001$ & 14.9 & .007 & 9.6 & .009 & 4.5 & .040 \\
\hline Feature priming (FP) & 6.8 & .035 & $<1.0$ & .821 & 10.2 & .015 & 9.1 & .019 & 8.7 & .025 & 3.8 & .093 & $<1.0$ & .612 \\
\hline Response priming (RP) & $<1.0$ & .837 & 5.8 & .047 & $<1.0$ & .896 & $<1.0$ & .989 & $<1.0$ & .444 & $<1.0$ & .406 & $<1.0$ & .740 \\
\hline $\mathrm{FP} \times \mathrm{SS}$ & 4.0 & .044 & $<1.0$ & .650 & 5.5 & .026 & 1.4 & .273 & 1.8 & .225 & $<1.0$ & .672 & $<1.0$ & .466 \\
\hline $\mathrm{FP} \times \mathrm{RP}$ & 22.5 & .002 & 3.4 & .108 & 23.5 & .002 & 6.1 & .043 & $<1.0$ & .714 & 1.6 & .242 & 7.3 & .030 \\
\hline
\end{tabular}

Note-The results are all Greenhouse-Geisser corrected and are depicted separately for manual response times (RTs) and error scores, as well as the mean target fixation latencies, the mean percentage of first saccades on the target and their respective latencies, and finally, the mean fixation durations on a distractor and the target. The two-way interaction between set size and response repetition and the three-way interaction were excluded because they never reached significance.

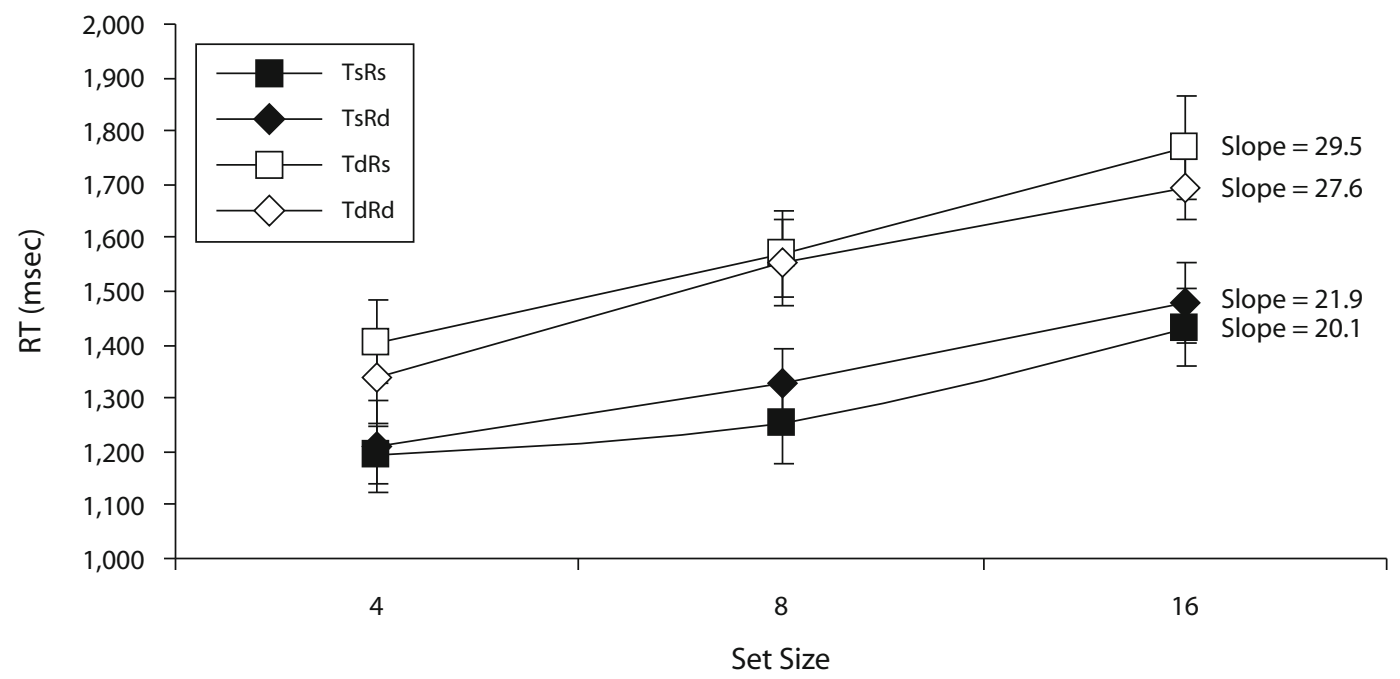

Figure 2. Mean response times (RTs), depicted separately for the different set sizes and the different intertrial contingencies: TsRs, both the target and the response were the same as in the previous, $n-1$ trial; TsRd, target same, response different; TdRs, target different, response same; TdRd, target different, response different. Error bars represent $1 S E M$.

both the target feature from the previous trial and the response were repeated ("TsRs" = target same, response same) do not differ strongly from trials on which only the response was changed ("TsRd" = target same, response different). However, the two graphs differ visibly depending on whether the target feature was changed, as compared with the previous trial, regardless of whether the response also changed ("TdRd" = target different, response different) or whether the response was repeated ("TdRs" = target different, response same).

The statistical analyses showed that correspondingly, feature priming had a significant main effect (see Table 1), since mean RTs were about 239 msec shorter on target feature repetition trials $(M=804 \mathrm{msec})$ than on different-targetfeature trials $(M=1,043 \mathrm{msec})$. RTs for response repetition trials and different-response trials differed slightly with respect to whether the target-defining feature was repeated or changed: When the target-defining feature was repeated, repeating the response-indicative item slightly speeded RTs ("TsRs"), as compared with different-response trials ("TsRd"). In contrast, when the target-defining feature changed, repeating the response slightly impaired performance, as is indicated by higher RTs on response repetition trials ("TdRs") than on different-response trials ("TdRd"). These exactly opposing effects of repeating the response in target repetition and different-target trials resulted in a significant interaction between feature priming and response priming effects (see Table 1).

Table 2

Mean Percentages of Error Scores (With Standard Errors of the Means) for the Experimental Conditions

\begin{tabular}{|c|c|c|c|c|c|c|c|c|}
\hline \multirow{2}{*}{$\begin{array}{c}\text { Set } \\
\text { Size }\end{array}$} & \multicolumn{2}{|c|}{ TsRs } & \multicolumn{2}{|c|}{ TsRd } & \multicolumn{2}{|c|}{ TdRs } & \multicolumn{2}{|c|}{$\mathrm{TdRd}$} \\
\hline & $\mathrm{PE}$ & $S E M$ & $\mathrm{PE}$ & $S E M$ & $\mathrm{PE}$ & $S E M$ & $\mathrm{PE}$ & SEM \\
\hline 4 & 2.50 & 0.60 & 2.58 & 1.14 & 3.71 & 1.77 & 0.53 & 0.35 \\
\hline 8 & 0.97 & 0.70 & 1.57 & 0.64 & 2.93 & 2.04 & 1.51 & 1.02 \\
\hline 16 & 3.04 & 1.83 & 2.48 & 1.86 & 4.05 & 1.47 & 1.07 & 0.71 \\
\hline
\end{tabular}

Note-TsRs, target and response-defining features were repeated (target same, response same); TsRd, only the target was repeated (target same, response different); TdRs, only the response was repeated (target different, response same); TdRd, both target-defining and response-defining features changed (target different, response different), relative to the previous, $n-1$ trial. 
More important, priming also significantly modulated search efficiency, as reflected in the significant feature priming $\times$ set size interaction (see Table 1). Separate $2 \times 2$ ANOVAs comprising intertrial contingencies of the target feature and the response-indicative feature revealed that repeating the target feature significantly reduced the slope of the set size function $[F(1,7)=5.7, p=.048]$ but did not affect its $y$-intercept $[F(1,7)=2.8, p=.137]$. In contrast, repeating the response-indicative item modulated neither the slope nor the $y$-intercept of the search function (all $p \mathrm{~s}>.1$ )

However, repeating the target feature only moderately improved search efficiency: Search rates (see Figure 2B, "slopes") were reduced only from about $28 \mathrm{msec} /$ item on different-target trials to about $20 \mathrm{msec} /$ item on target feature repetition trials. The slopes in each of the four conditions were still significantly different from zero (all $p$ s $<$ .001 ; two-sided $t$ test). Thus, the present results certainly do not warrant the conclusion that priming can generally account for efficient conjunction search (e.g., Geyer et al., 2006; Kristjánsson et al., 2002). However, the finding that repeating the target feature improves search efficiency suggests that priming affects processes concerned with attentional guidance. This interpretation, however, needs to be substantiated by the eye movement data.

\section{Eye Movement Data}

The results of the $3 \times 2 \times 2$ ANOVA are shown in Table 1. Figure 3A shows the mean target fixation latencies in each of the conditions. The target fixation latency is the time from display onset to when the eyes first fixate the target on each trial. Thus, the target fixation latencies can be viewed as a correlate to the mean RTs on the level of eye movements.

As can be seen in Figure 3A, the mean target fixation latencies also closely resemble the result pattern for the manual RTs, with latencies increasing with increases in set size and shorter target fixation latencies on target feature repetition trials than on different-target trials (see also Table 1). Moreover, repeating the target feature significantly reduced the set size effect, as is shown by the significant interaction between feature priming and set size (see Table 1). To examine whether priming affects processes that guide attention to the target, by varying the probability of target selection on a trial-by-trial basis, the percentage of first fixations on the target on each trial were analyzed. Figure 3B shows that the percentage of first saccades that directly went to the target was highest in the set size 4 condition and declined with increasing set size, resulting in a significant main effect of set size (34\%, $20 \%$, and $20 \%$ in the set size 4,8 , and 16 conditions, respectively; see also Table 1). Moreover, there was also a significant priming effect: Repeating the target-distractor features across trials significantly enhanced the percentage of first saccades on the target, from $19 \%$ on switch trials to $32 \%$ on repetition trials (see Table 1). However, repeating the target feature from the previous trial did not significantly interact with the set size effect (see Table 1). Instead, the feature priming $\times$ response priming interaction reached significance, showing that repeating the re- sponse increased the mean percentage of first saccades on the target only when the target-defining feature was repeated too (mean difference $=4.2 \%$ ) but decreased the mean percentage of first saccades on the target when the target-defining feature changed (mean difference $=$ $-4.2 \%$; see also Table 1 ).

We also analyzed the latencies of the first saccades on the target, to exclude the possibility that the previous results were due to a speed-accuracy trade-off- that is, to exclude the possibility that the higher percentage of correct saccades (e.g., on repetition trials) were really due to the fact that the observers took more time to identify the target prior to executing the saccade (see Figure 3C and Table 1). The results showed that saccade latencies increased with increases in the set size, with latencies of $378 \mathrm{msec}$ in the set size 4 condition, $459 \mathrm{msec}$ in the set size 8 condition, and $579 \mathrm{msec}$ in the set size 16 condition. Moreover, repeating the target feature led to shorter saccade latencies $(M=453 \mathrm{msec})$ than did changing the target from the previous trial $(M=503 \mathrm{msec})$. Thus, interpretation of the results is not complicated by a speedaccuracy trade-off.

Next, the distractor fixation durations and target fixation durations were submitted to the same analyses. Figure $3 \mathrm{D}$ depicts the mean distractor fixation durationsthat is, the mean durations for which the eyes remained fixated on one of the distractor items. Somewhat unexpectedly, the analyses showed a significant main effect of set size, because distractor fixation durations decreased with increasing number of distractor items, yielding 245-, 220-, and 214-msec fixation durations in the set size 4, 8, and 16 conditions, respectively (see Table 1). However, feature priming did not significantly modulate the distractor fixation durations, since the fixation durations were comparable in repetition trials $(M=222 \mathrm{msec})$ and switch trials $(M=230 \mathrm{msec})$.

The mean target fixation durations (i.e., the duration for which the eyes remained fixated on the target) are depicted in Figure 3E. The target fixation durations also slightly decreased with increasing set size, yielding fixation durations of 440,433 , and $424 \mathrm{msec}$ in the set size 4,8 , and 16 conditions, respectively (see Table 1 ). There were no differences between target feature repetition trials and different-target trials (see Table 1). However, repeating the response significantly interacted with priming of the target-defining feature, showing that repeating the response led to shorter mean target fixation durations only when the target-defining feature was also repeated (mean difference $=-14 \mathrm{msec}$ ), whereas it had the reverse effect when the target-defining feature had switched (mean difference $=17 \mathrm{msec}$ ) .

Taken together, the results suggest that priming in conjunction search affects processes occurring during visual search: When the target, and distractor features from the previous trial are repeated, the target can be found more quickly, and a significantly higher percentage of first saccades is directly guided to the target. In contrast, priming does not modulate processes after selection of the target or after selection of one of the distractors. This result pattern closely resembles previous results obtained in a pop-out 


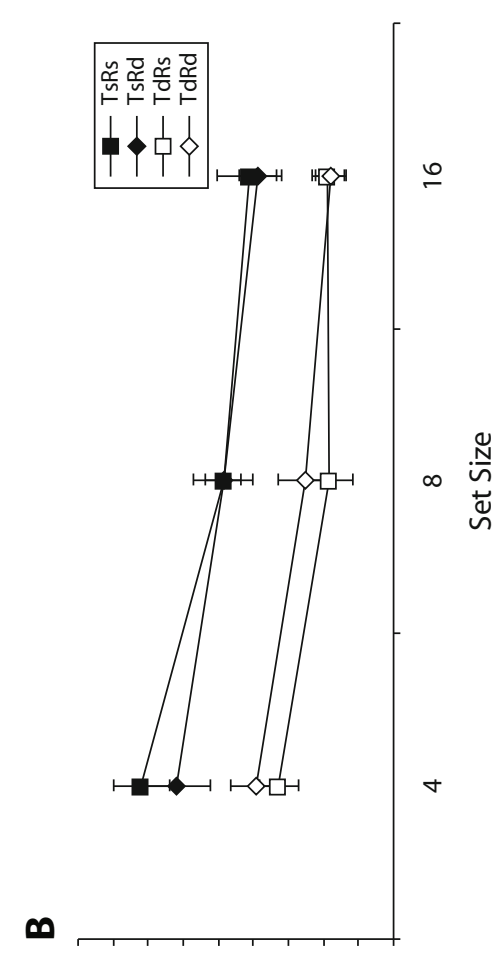

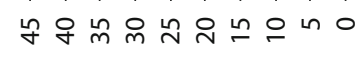

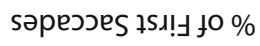

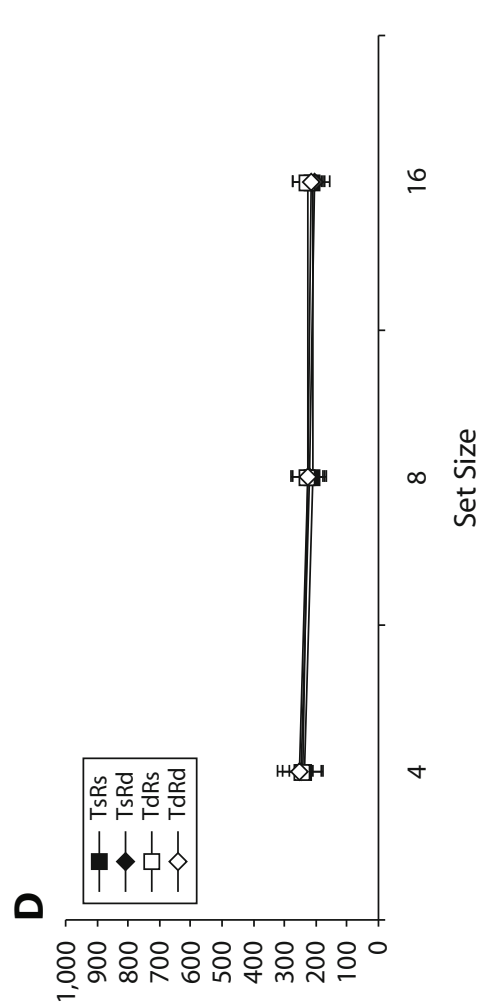

(गəsw)

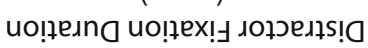

:

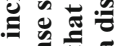

政

政

政

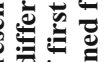

둥

䉼

은

可

:

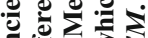

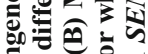

政

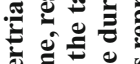

$\stackrel{6}{-}$ $=5$

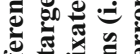

ชี

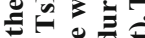

כ)

范

$\infty$ 芩

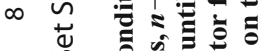

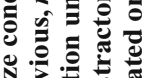

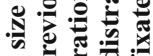

远家

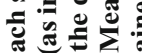

है

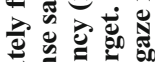

政

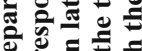

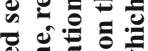

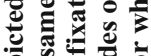

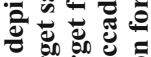

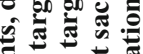

$\infty$ (J)

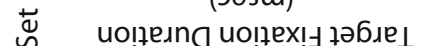


search task (e.g., Becker, 2008b) and suggests that priming in conjunction search modulates the same processes as those in pop-out search.

\section{Cumulative Priming Effects}

To assess whether cumulative priming effects will lead to further reductions in the set size effect, data from the repetition trials were separately analyzed according to their position in a sequence of same target-distractor displays. As can be seen in Figure 4A, RTs generally declined with number of consecutive repetitions. However, the set size effect does not seem to be reduced with increasing repetitions, as can be seen by the consistently large differences between the set size 4,8 , and 16 conditions.

Statistical analyses confirmed this impression: A $3 \times 5$ ANOVA with the variables of set size (4 vs. 8 vs. 16) and repetition factor (1 switch, 1-4 repetition trials) computed over the mean RTs showed a significant main effect of set size $[F(2,14)=52.7, p<.001]$ and of repetition $[F(4,28)=7.3, p=.009]$ but no significant reduction of the set size effect with increasing repetitions $(F<1)$. The set size effect differed only between switch trials and the first repetition trials $[F(2,14)=6.8, p=.034]$, but not across the different repetition trials (all $p \mathrm{~s}>.4$ ).

The same analysis computed over the mean target fixation latencies, or VRTs, showed similar results (see Figure 4B): There was a significant main effect of set size $[F(2,14)=38.3, p<.001]$ and of repetition $[F(4,28)=$ $9.3, p=.004]$ but no significant reduction of the set size effect with increasing repetitions $(F<1)$. The set size effect again differed only between switch trials and the first repetition $[F(2,14)=5.4, p=.019]$, but not between the
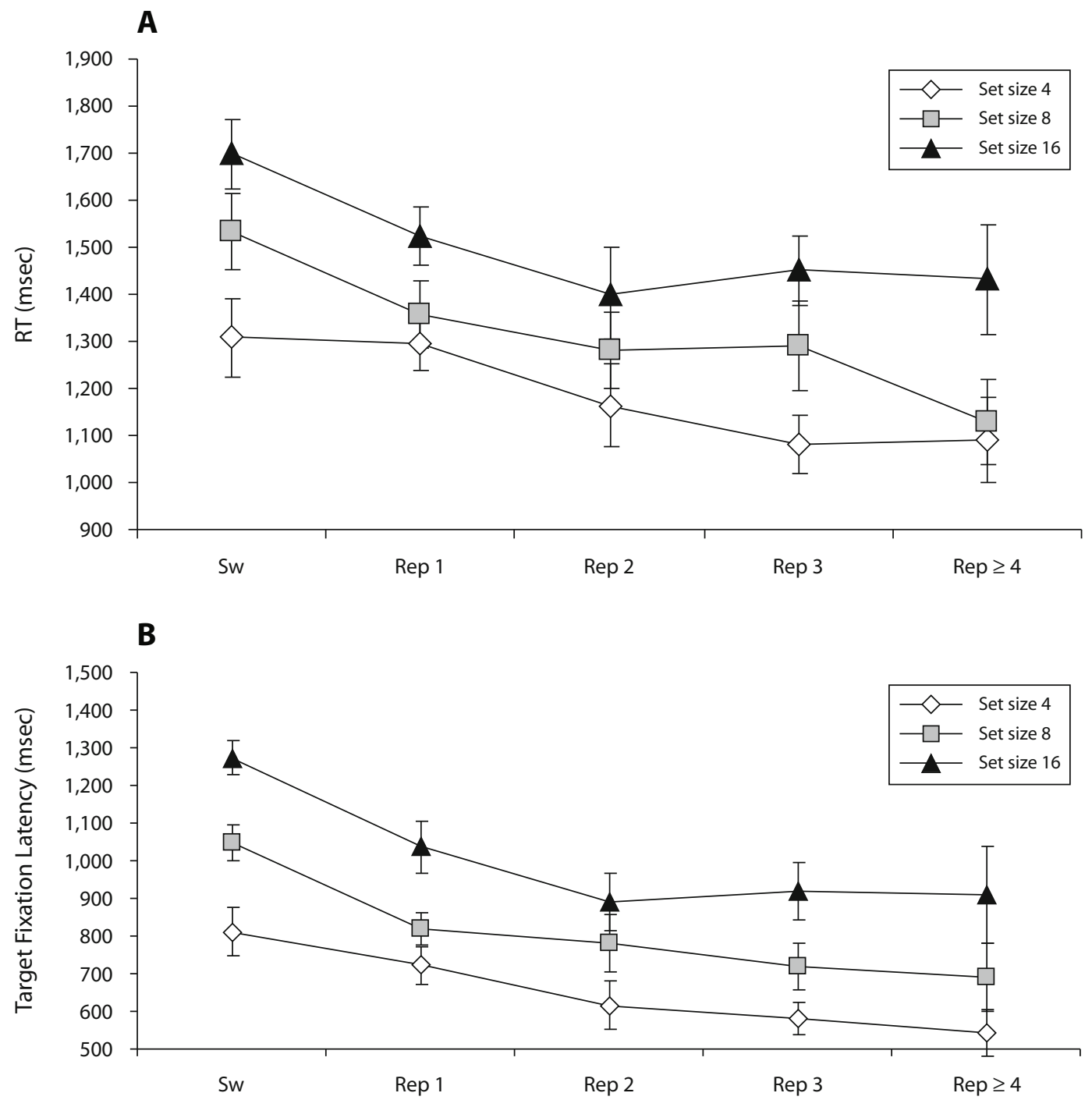

Figure 4. Cumulative priming effects depicted separately for each set size condition and separated according to the order of a trial in a sequence of trials: From left to right, mean performance on switch trials ("Sw") and on first repetition ("Rep 1"), second repetition ("Rep 2), third repetition ("Rep 3"), and more than three repetitions in a sequence ("Rep $\geq 4$ "). (A) Mean response times (RTs). (B) Mean target fixation latencies. Error bars represent $1 S E M$. 


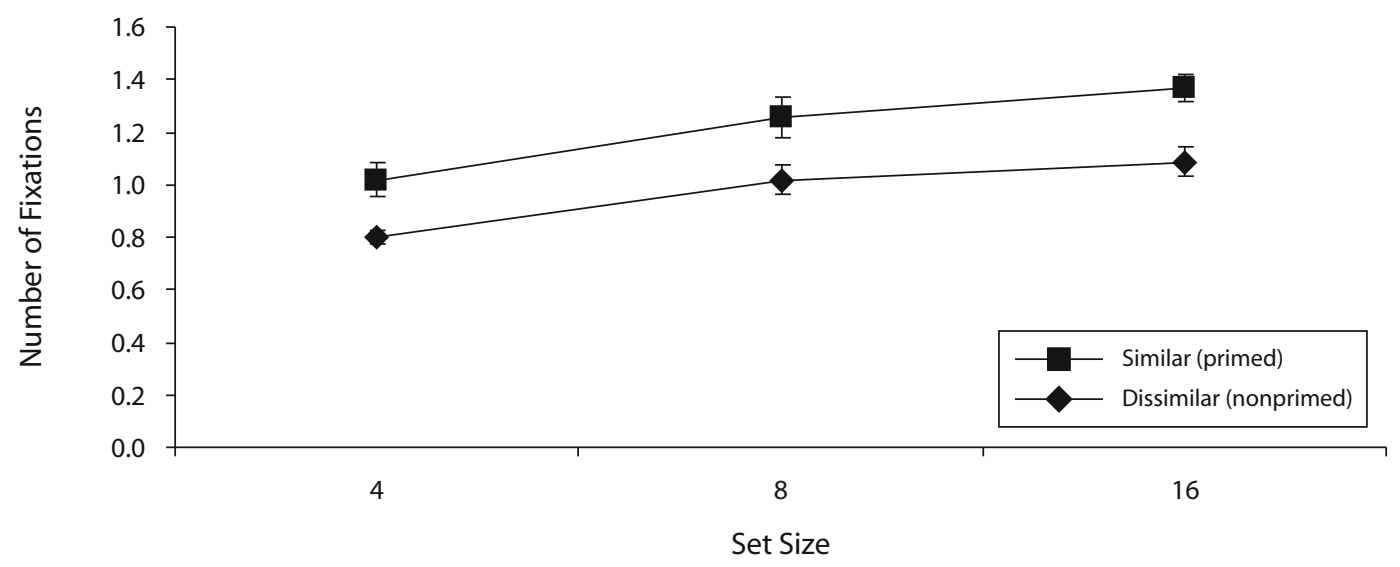

Figure 5. Mean number of fixations on the distractors, separated according to the different set size conditions and whether the fixation occurred on distractors possessing the same color as the target previous, $n-1$, trial ("similar") or whether a different color distractor was fixated ("dissimilar"). Error bars represent 1 SEM.

different repetition trials (all $p \mathrm{~s}>.3$ ). The percentages of first fixations on the target were not analyzed for cumulative priming effects because the amount of data was insufficient for statistical analyses.

The results from the cumulative analysis indicate that successive repetitions of the target-distractor features across trials reduce only the mean search times but do not reduce the slope of the set size function. Repeating the target once reduced the mean slopes from $29 \mathrm{msec} /$ item to $20 \mathrm{msec} / \mathrm{item}$, but further repetitions yielded slopes of 18 , 26 , and $25 \mathrm{msec} / \mathrm{item}$, respectively. The slight and nonsignificant rise in the set size effect might be due to expectation effects: Since the target-distractor features were drawn randomly on each trial, the expectation value for successive repetitions was 2 . Therefore, it is possible that after two repetitions, the participants expected a change in the features of target and/or distractors (gambler's fallacy), which again slightly increased the search slope. However, further research is necessary to investigate this possibility in greater detail.

More important, this result pattern indicates that further repetitions of the features of target and distractors do not further reduce the slope of the set size function. This effectively excludes the possibility that efficient conjunction search can be explained by cumulative priming effects.

\section{Analysis of Subgroup Selections}

The question of whether priming in conjunction search accords with a feature-weighting mechanism or one of the mechanisms proposed by the alternative accounts (memory retrieval, grouping, or stimulus encoding) was assessed by separately evaluating the number of fixations on red and green distractor items. As can be seen in Figure 5, repeating the target and distractor features led to significantly more frequent fixations on distractor items that had the same color as the target on the previous, $n-1$ trial. A $3 \times$ 2 ANOVA comprising the variables of set size (4 vs. 8 vs. 16) and similarity of the target-defining feature (distractor similar vs. dissimilar to previous target color) calculated over the mean number of distractor fixations showed, first, a significant main effect of set size $[F(2,14)=43.1, p<$ $.001]$, reflecting that increasing the set size from 4 to 8 and 16 items led to increases in the mean number of fixations of $0.9,1.1$, and 1.2, respectively. Second and more important, the results showed that distractors that had the same color as the target on the previous trial were significantly more frequently selected $(M=1.2)$ than distractors that did not share the color of the previous target $(M=0.9)$ $[F(1,7)=6.4, p=.039]$.

The same analysis was computed over the distractor fixation durations, to assess whether feature weighting additionally modulates the durations for which the eyes remain fixated on each distractor. However, the results of the same ANOVA showed only an inverse set size effect, reflecting that distractor fixation durations decreased with increases in the set size, measuring 252,223, and $216 \mathrm{msec}$ in the set size 4,8 , and 16 conditions, respectively $[F(2,14)=9.3, p=.013]$. Specifically, there was no significant effect of priming $[F(1,7)=1.4, p=.283]$. As can be seen in Figure 6, fixations on distractors sharing the color of the previous target were of a length $(M=$ $225 \mathrm{msec}$ ) comparable to that for fixations on distractors that had a different color $(M=235 \mathrm{msec})$.

Taken together, the results indicate that selection of the target on the current trial leads to selective prioritization of same-colored items on the next trial(s). These results are not in line with the claim that priming affects only preparatory processes that modulate memory retrieval, stimulus encoding, or grouping. Instead, the results support a feature-weighting account of priming.

\section{DISCUSSION}

The present study yielded several interesting results. First, this is the first study to demonstrate that priming modulates search efficiency in conjunction search. Contrary to previous studies, the present eye movement study revealed that repeating the features of target and 


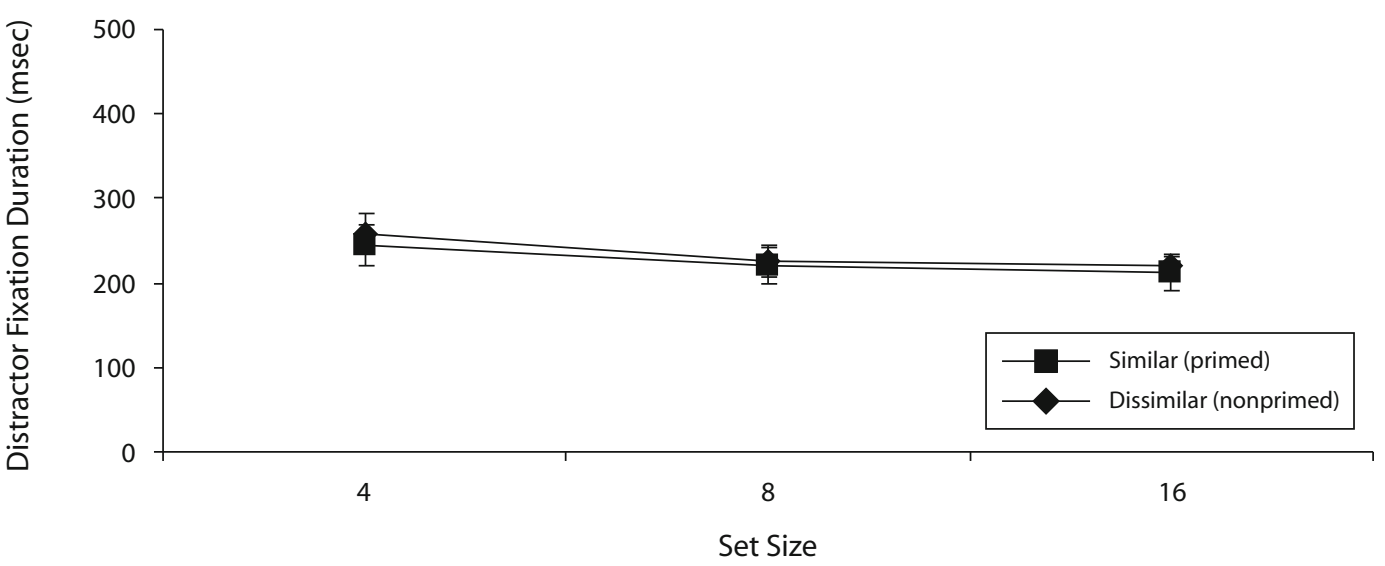

Figure 6. The mean fixation durations on the distractors (i.e., the durations for which the gaze remained fixated on any of the distractors), depicted separately for the different set sizes and according to whether the fixation occurred on distractors possessing the same color as the target in the previous, $n-1$ trial ("similar") or whether they occurred on a different-colored distractor ("dissimilar"). Error bars represent 1 SEM.

distractors in conjunction search enhances search efficiency. Priming modulates not only the base RTs, but also the slope of the search function, or the set size effect, when the target features are clearly defined and participants cannot profit from subset search strategies. This result pattern is incompatible with all explanations of the priming effect that predict a reduction only in the $y$-intercept or the base RTs on repetition trials (e.g., Hillstrom, 2000).

Second, however, the results do not lend support to the hypothesis that priming can account for efficient conjunction search (e.g., Geyer et al., 2006). Even on trials on which the target-distractor features were repeated, search remained inefficient, with slopes of around $20 \mathrm{msec} /$ item. Moreover, when the target-distractor features were repeated consecutively for several trials in a row, search rates were still significantly different from zero, and performance did not reach the range of $0-10 \mathrm{msec} /$ item usually considered as reflecting efficient search (e.g., Treisman \& Sato, 1990).

Third, concerning the question of whether priming affects the search process or postselectional processes commencing after the search target has been found, the results were clear-cut: Repeating the features of target and distractors led to faster visual selection of the search target (VRTs) but did not modulate postselectional processes such as decisional or response-related processes occurring after selection. The priming effect in the VRTs was consistently of the same magnitude as that in the mean RTs, leaving no room for contributions of processes that commence after target selection.

Fourth, priming modulated processes concerned with guiding attention to the target: This was reflected in the significantly higher percentage of first fixations on the target on repetition than on switch trials and in the finding that distractors that had the same color as the target in the previous, $n-1$ trial were more frequently selected than distractors that were of a different color. These results are fully consistent with a feature-weighting ac- count and do not support alternative explanations that propose that priming modulates early perceptual processes that are unrelated to attentional guidance (e.g., retrieval of episodic memory traces, grouping or parallel encoding of all features in the display). Although the present results certainly do not rule out the possibility that these processes might additionally contribute to the priming effect, they indicate that priming plays a more direct role in guiding attention to the target than is suggested by the alternative views.

Taken together, the results from the present study suggest that priming in conjunction search operates on the same processes as priming of pop-out and, thus, is not fundamentally different from priming in pop-out search.

\section{Set Size Effects and Search Efficiency}

In visual search, it is commonly assumed that changes in the probability of selecting the target or distractor should modulate the RT $\times$ set size function. This holds because the RT $\times$ set size function is presumably determined by the number of distractors that are selected on each trial and the time it takes to reject a distractor. All other things being equal, all processes that enhance the probability that the target will be selected should therefore lead to a reduction in the number of distractors selected and should reduce the slope of the search function. With regard to the priming effect, this assumption led several different authors to conclude that priming does not change the probability of selecting the target but, instead, modulates the time needed to encode stimuli, group them, or retrieve attentional control settings or a priority rule.

However, in the present study, we found strong evidence for the hypothesis that priming changes the probability of selecting the target on a trial-by-trial basis: For instance, repeating the target-distractor features greatly enhanced the percentage of first fixations on the target (see Figure 3B). Contrary to this large effect in the probability of selecting the target as the first item in the display, repeti- 
tions only moderately enhanced search efficiency: As can be seen in Figures 2B and 3A, the main effect of priming on mean RTs and VRTs was considerably stronger than the priming $\times$ set size interaction (see also Table 1). The finding that the RT $\times$ set size interaction was only moderately affected despite large changes in the probability of selecting the target suggests that the RT $\times$ set size function is probably not the most sensitive indicator of search efficiency. The results do not allow pinpointing why the $\mathrm{RT} \times$ set size function is probably not the most sensitive measure for changes in search efficiency. Nevertheless, the results suggest that processes that modulate the probability that the target will be selected are not expressed to the same degree in the RT $\times$ set size function.

These results call into question the assumption that all processes that are rightly classed as attentional processes should reduce the slope of the RT $\times$ set size function. Correspondingly, one should probably refrain from drawing substantial conclusions from the observation that certain manipulations do not reduce the slope of the search function, but only the $y$-intercept (e.g., Kunar et al., 2007). The results of the present study suggest that measuring the eye movements might be a more direct way of investigating whether certain manipulations affect search efficiency than is the RT $\times$ set size function.

With regard to the question of why the RT $\times$ set size function might not always be a reliable indicator for attentional processes, it can be speculated that there are processes opposing the set size function. It is interesting to note that, in the present study, the mean fixation durations on the target and on each distractor item were inversely related to set size: Mean target and distractor fixation durations decreased with increases in the number of distractors.

Several different explanations may conceivably account for this inverse set size effect. First, it is possible that prolonged fixations on distractors in sparse displays might reflect difficulties in attentional control, so that it becomes more difficult to discern a candidate target item and allocate attention to it. In this case, the fixation durations would be related not to perceptual-processing difficulty but to difficulties in processes that single out the next to-be-attended item and guide attention to it. Although possible, this explanation is at odds with the observation that the latencies of the first saccades increased with increasing set size.

Second, the inverse set size effect might be due to within-display repetition facilitation effects, so that perceptual identification of a feature commences more quickly when previously, similar items have been selected and subjected to the same analysis. Since the number of distractor fixations increases with increases in set size, the probability that perceptual identification processes can profit from repetition facilitation increases with increasing set size. Alternatively, or additionally, it is conceivable that participants lower their criterion for terminating a fixation with increasing set size, in order to keep search times at a reasonable level. ${ }^{3}$ On the basis of the present study, it cannot be decided which of the above-mentioned hypotheses account for decreasing fixation durations at increasing set sizes. Further research is needed to explore this question in more detail.

\section{Feature Weighting and Search Efficiency}

The present results unequivocally support a featureweighting account of the priming effect in conjunction search, which proposes that priming changes the attentiondriving capacity of all features on a trial-by-trial basis. However, this interpretation might also be criticized. As Hillstrom (2000) pointed out, if priming were indeed based on feature weighting, repeating the target-distractor features across trials should lead to strong reductions in the set size effect. In contrast, in the present study, we found only rather moderate reductions of the set size effect, which were around $10 \mathrm{msec} /$ item.

On the other hand, it can also be argued that a featureweighting view is very compatible with the finding that priming does not completely eliminate the set size effect. At least in a conjunction search task, a feature-weighting account would not necessarily predict large reductions in the set size effect when the target-distractor features are repeated. This holds because, in conjunction search, the target always shares one of its features with the distractors; thus, enhancing the activation signals of the target features will also enhance the activation signals of (statistically) half of all distractor items. Although the target will probably profit most from repetitions, the activation signals from the target will have to compete with simultaneously enhanced noise from the distractors. Thus, repeating the target in conjunction search probably cannot enhance the signal-to-noise ratio of the target to such an extent that search becomes efficient. From the feature-weighting account, it thus does not follow that repeating the target in conjunction search should lead to dramatic reductions of the set size effect. With this, the feature-weighting view seems to be very compatible with the observation that repeating the target across trials did not lead to efficient search, or a zero set size function.

Moreover, the feature-weighting account can probably also account for the differences in the magnitude of the priming effect observed across different studies. As was mentioned above, the rather small feature-priming effect observed in the studies of Kristjánsson et al. (2002) and Geyer et al. (2006), amounting to 20 and $40 \mathrm{msec}$, respectively, might be due to the fact that the target never changed its color across trials. Presumably, the priming effect rested only on changes in the orientation of the target and distractor features. When both target features of a conjunctively defined target changed across trials, as in the study of Hillstrom (2000) and the present study, priming effects were much larger-between 440 and $240 \mathrm{msec}$, respectively. These results are consistent with the claim of the feature-weighting view that changing two different target-defining features (e.g., orientation and color) results in additive switch costs. On the featureweighting view, activation signals of different feature values are stored separately, and the weights for each feature are incremented and decremented independently of each other. If weighting occurs independently for different features, it follows that changing two features together should lead to additive switch costs. Taken together, the featureweighting account could thus account in part for the large differences in the magnitude of the priming effect across 
different studies, in which only one versus two target features varied across trials (e.g., Maljkovic \& Nakayama, 1994, 1996).

The finding that priming effects in Hillstrom's (2000) study were larger than those in the present study might additionally be due to Hillstrom's using a very difficult search task, in which the search target was not easily discriminable from the distractors (target and distractors could be either pink or red and textured or untextured). As Hillstrom remarked, this task was so difficult that it could be performed only when the target color was known in advance to the participants. Since priming effects have been reported to be larger with increasing task difficulty (or less discriminable targets), the differences in the discriminability of the target and distractor stimuli might account for the larger priming effect in Hillstrom's study than those observed in the present study (Meeter \& Olivers, 2006; Olivers \& Meeter, 2006). However, further research is needed to explore the impact of search difficulty on priming in conjunction search in more detail.

\section{Can Attention Be Guided by Holistic Target Representations?}

The present results, however, also appear to be at variance with the claim of the feature-weighting view, that weights for different target features are incremented and decremented independently of each other. In the present conjunction search task, priming of the target-defining feature interacted with priming of the response-indicative feature: Mean RTs and VRTs were shortest when both features were repeated or switched together, whereas repeating only one of these features resulted in elongated RTs and VRTs.

The same interaction between repeating the targetdefining feature and the response-indicative feature has already been reported in previous studies (Hillstrom, 2000; Huang, Holcombe, \& Pashler, 2004). However, as was pointed out in these studies, this result pattern is incompatible with the view that multiple features of the target are weighted independently of each other (Hillstrom, 2000; Huang et al., 2004). Instead, it suggests that the benefits of repeating the response-indicative feature critically depend on whether the target-defining feature is repeated or switched.

At a first glance, this result pattern seems to indicate that weightings can also pertain to conjunctions of features, so that attention could also be guided by holistic target representations that contain information about feature conjunctions (see, e.g., Huang et al., 2004; Kristjánsson, 2006b). However, this view is at odds with the main hypothesis of several influential visual search theoriesmost notably, perhaps, feature integration theory (FIT; e.g., Treisman \& Gelade, 1980; Treisman \& Sato, 1990). According to this theory, attention can generally be guided only by simple or primitive features, whereas attentional processing is necessary to bind distinct features together and to recognize feature conjunctions.

In previous contributions, it was therefore hypothesized that the response priming effect might be generally due to processes located at the postselectional stage, such as decision- or response-related processes (Becker, 2008b; Hillstrom, 2000; Huang et al., 2004). However, the present results do not support the view that the response-priming effect resides in postselectional processes. As can be seen in Figure 3B and Table 1, the interaction between repeating the target-defining and response-related feature already occurred in the percentage of first fixations on the target. This result pattern suggests that intertrial contingencies of both the target-defining and the response-indicative features can affect attentional guidance to the target, contrary to previous explanations and, also, at odds with the view that attention cannot be guided by feature conjunctions (e.g., Treisman \& Gelade, 1980; Treisman \& Sato, 1990). Admittedly, guidance of attention by conjunctively defined targets cannot be ascertained with absolute certainty in the present study, because eye movements are usually preceded by covert attention shifts but cannot be directly equated with covert attention shifts. Therefore, it is possible that prior to shifting the gaze to a certain location, covert attention had already been deployed to the location, which allowed identifying both the target-defining feature and the response-indicative feature and provided the required information for the first eye movement.

On the other hand, the possibility that covert attention can be guided by feature conjunctions should not be dismissed as preposterous. As was mentioned in the introduction, there have been several studies reporting that search for a conjunctively defined target can proceed efficiently - that is, with search slopes below $10 \mathrm{msec} /$ item (e.g., Wang et al., 2005). Assuming that attention can be directed by representations that contain information about feature conjunctions might present the most parsimonious account of these findings. The challenge for the future will be to determine the factors allowing efficient conjunction search.

\section{AUTHOR NOTE}

We thank James E. Hoffman, Árni Kristjánsson, and two anonymous reviewers for their constructive and insightful comments and suggestions on earlier drafts of the manuscript. Correspondence concerning this article should be addressed to S. I. Becker, School of Psychology, University of Queensland, McElwain Building (24A), St. Lucia, QLD 4072, Australia (e-mail: s.becker@psy.uq.edu.au).

\section{Note-Accepted by the previous editorial team, when Thomas H. Carr was Editor}

\section{REFERENCES}

BECKER, S. I. (2007). Irrelevant singletons in pop-out search: Attentional capture or filtering costs? Journal of Experimental Psychology: Human Perception \& Performance, 33, 764-787.

BECKER, S. I. (2008a). Can intertrial effects of features and dimensions be explained by a single theory? Journal of Experimental Psychology: Human Perception \& Performance, 34, 1417-1440.

BECKER, S. I. (2008b). The mechanism of priming: Episodic retrieval or priming of pop-out? Acta Psychologica, 127, 324-339.

BECKER, S. I. (2008c). The stage of priming: Are intertrial repetition effects attentional or decisional? Vision Research, 48, 664-684.

Deubel, H., \& Schneider, W. X. (1996). Saccade target selection and object recognition: Evidence for a common attentional mechanism. Vision Research, 36, 1827-1837. 
Duncan, J., \& Humphreys, G. W. (1989). Visual search and stimulus similarity. Psychological Review, 96, 433-458.

D’Zmura, M. (1991). Color in visual search. Vision Research, 31, 951966.

Egeth, H. E., Virzi, R. A., \& Garbart, H. (1984). Searching for conjunctively defined targets. Journal of Experimental Psychology: Human Perception \& Performance, 10, 32-39.

Found, A., \& Müller, H. J. (1996). Searching for unknown feature targets on more than one dimension: Investigating a "dimensionweighting" account. Perception \& Psychophysics, 58, 88-101.

Geyer, T., Müller, H. J., \& Krummenacher, J. (2006). Cross-trial priming in visual search for singleton conjunction targets: Role of repeated target and distractor features. Perception \& Psychophysics, 68, 736-749.

Goolsby, B. A., \& SuZuKi, S. (2001). Understanding priming of colorsingleton search: Roles of attention at encoding and "retrieval." Perception \& Psychophysics, 63, 929-944.

Hillstrom, A. P. (2000). Repetition effects in visual search. Perception \& Psychophysics, 62, 800-817.

Huang, L., Holcombe, A. O., \& Pashler, H. (2004). Repetition priming in visual search: Episodic retrieval, not feature priming. Memory \& Cognition, 32, 12-20.

HuAnG, L., \& PASHLER, H. (2005). Attention capacity and task difficulty in visual search. Cognition, 94, B101-B111.

Iтті, L., \& Косн, C. (2000). A saliency-based search mechanism for overt and covert shifts of visual attention. Vision Research, 40, 14891506.

Kaptein, N. A., Theeuwes, J., \& VAn der Heidden, A. H. C. (1995). Search for a conjunctively defined target can be selectively limited to a color-defined subset of elements. Journal of Experimental Psychology: Human Perception \& Performance, 21, 1053-1069.

KRISTJÁnsSON, Á. (2006a). Rapid learning in attention shifts: A review. Visual Cognition, 13, 324-362.

KRISTJÁNSSON, Á. (2006b). Simultaneous priming along multiple feature dimensions in a visual search task. Vision Research, 46, 2554-2570.

Kristú́nsson, Á., Wang, D., \& Nakayama, K. (2002). The role of priming in conjunctive visual search. Cognition, 85, 37-52.

Kunar, M. A., Flusberg, S., Horowitz, T. S., \& Wolfe, J. M. (2007). Does contextual cuing guide the deployment of attention? Journal of Experimental Psychology: Human Perception \& Performance, 33, 816-828.

Leonard, C. J., \& Egeth, H. E. (2008). Attentional guidance in singleton search: An examination of top-down, bottom-up, and intertrial factors. Visual Cognition, 16, 1078-1091.

Maljkovic, V., \& Nakayama, K. (1994). Priming of pop-out: I. Role of features. Memory \& Cognition, 22, 657-672.

Maljkovic, V., \& Nakayama, K. (1996). Priming of pop-out: II. The role of position. Perception \& Psychophysics, 58, 977-991.

McLeod, P., Driver, J., \& Crisp, J. (1988). Visual search for a conjunction of movement and form is parallel. Nature, 332, 154-155.

McPeek, R. M., Maljkovic, V., \& Nakayama, K. (1999). Saccades require focal attention and are facilitated by a short-term memory system. Vision Research, 39, 1555-1566.

MeEter, M., \& Olivers, C. N. L. (2006). Intertrial priming stemming from ambiguity: A new account of priming in visual search. Visual Cognition, 13, 202-222.

Müller, H. J., Heller, D., \& Ziegler, J. (1995). Visual search for singleton feature targets within and across feature dimensions. Perception \& Psychophysics, 57, 1-17.

NaKayama, K., \& Silverman, G. H. (1986). Serial and parallel processing of visual feature conjunctions. Nature, 320, 264-265.
Olivers, C. N. L., \& Humphreys, G. W. (2003). Attentional guidance by salient feature singletons depends on intertrial contingencies. Journal of Experimental Psychology: Human Perception \& Performance, 29, 650-657.

Olivers, C. N. L., \& Meeter, M. (2006). On the dissociation between compound and present/absent tasks in visual search: Intertrial priming is ambiguity driven. Visual Cognition, 13, 1-28.

PALMER, J. (1995). Attention in visual search: Distinguishing four causes of a set-size effect. Current Directions in Psychological Science, 4, 118-123.

Rauschenberger, R., \& Yantis, S. (2006). Perceptual encoding efficiency in visual search. Journal of Experimental Psychology: General, 135, 116-131.

Treisman, A. [M.] (1982). Perceptual grouping and attention in visual search for features and for objects. Journal of Experimental Psychology: Human Perception \& Performance, 8, 194-214.

Treisman, A. [M.] (1988). Features and objects: The Fourteenth Bartlett Memorial Lecture. Quarterly Journal of Experimental Psychology, 40A, 201-237.

Treisman, A. M. \& Gelade, G. (1980). A feature-integration theory of attention. Cognitive Psychology, 12, 97-136.

Treisman, A. [M.], \& Sato, S. (1990). Conjunction search revisited. Journal of Experimental Psychology: Human Perception \& Performance, 16, 459-478.

Treisman, A. [M.], \& Souther, J. (1985). Search asymmetry: A diagnostic for preattentive processing of separable features. Journal of Experimental Psychology: General, 114, 285-310.

Wang, D., KristuánsSon, Ả., \& NaKaYAma, K. (2005). Efficient visual search without top-down or bottom-up guidance. Perception \& Psychophysics, 67, 239-253.

WoLfe, J. M. (1994). Guided Search 2.0: A revised model of visual search. Psychonomic Bulletin \& Review, 1, 202-238.

Wolfe, J. M. (1998). Visual search. In H. Pashler (Ed.), Attention (pp. 13-73). Hove, U.K.: Psychology Press.

Wolfe, J. M., Cave, K. R., \& FranzeL, S. L. (1989). Guided search: An alternative to the feature integration model for visual search. Journal of Experimental Psychology: Human Perception \& Performance, 15, 419-433.

Wolfe, J. M., Friedman-Hill, S. R., Stewart, M. I., \& O'Connell, K. M. (1992). The role of categorization in visual search for orientation. Journal of Experimental Psychology: Human Perception \& Performance, 18, 34-49.

Yantis, S., \& EgEth, H. E. (1999). On the distinction between visual salience and stimulus-driven attentional capture. Journal of Experimental Psychology: Human Perception \& Performance, 25, 661-676.

\section{NOTES}

1. None of the three aforementioned studies contains a clear description of whether the displays consisted of an equal number of distractors of each subset or whether the number of distractors of each subset could vary. In the study of Kristjánsson et al. (2002), an example of the search display shows 9 red and 7 green elements in the set size 16 condition (see Figure 1 in Kristjánsson et al., 2002), whereas the example displays in Geyer et al. (2006) show equal numbers of red and green distractors, but these example displays are certainly not conclusive.

2. We are indebted to one of the reviewers for pointing this out to us.

3. We thank James E. Hoffman for pointing out this possibility.

(Manuscript received December 21, 2007; revision accepted for publication September 23, 2008.) 